\title{
$\mathrm{NCDs} / \mathrm{TiO}_{2}$ 复合材料的制备及其在太阳光下催化制氢的应用
}

\author{
魏 婕 ${ }^{1}$, 李雪冬 ${ }^{1}$, 王宏志 ${ }^{1}$, 张青红 ${ }^{2}$, 李耀刚 ${ }^{1}$
}

(东华大学 1. 材料科学与工程学院, 纤维材料改性国家重点实验室; 2. 先进玻璃制造技术教育部工程研究中心, 上 海 201620)

摘 要: 采用溶剂热法, 以乙腈为溶剂和葡萄糖为原料制备了粒径约 $4 \mathrm{~nm}$ 的氮掺杂碳量子点 NCDs。当激发波长从 $330 \mathrm{~nm}$ 增加到 $470 \mathrm{~nm}, \mathrm{NCDs}$ 水溶液发射光谱出现红移。随后, 将一定配比的 $\mathrm{NCDs} \mathrm{TiO}_{2}$ 及 $5 \mathrm{~mL}$ 超纯水超声混 合 $60 \mathrm{~min}$, 并在 $80^{\circ} \mathrm{C}$ 烘箱内陈化 $24 \mathrm{~h}, \mathrm{NCDs}$ 纳米颗粒成功地复合到 $\mathrm{TiO}_{2}\left(\mathrm{TiO}_{2}\right)$ 表面。该方法有效地拓宽了 $\mathrm{TiO}_{2}$ 吸 收光谱的范围, 并且减少了光生电子和空穴对, 从而增强了 $\mathrm{TiO}_{2}$ 的光催化制氢性能。实验结果表明: 投料比为 $m(\mathrm{NCDs}): m\left(\mathrm{TiO}_{2}\right)=15: 85$ 时, 以甲醇为牺牲剂的反应体系光催化制氢效果最好, 该复合材料具有一定的稳定性, 循 环三次使用后仍然有一定的光催化制氢性能。

关 键 词: NCDs; 碳量子点 $/ \mathrm{TiO}_{2}$ 复合材料; 太阳光催化制氢; 溶剂热合成

中图分类号: TQ127 文献标识码: A

\section{Nitrogen Doped Carbon Quantum Dots/Titanium Dioxide Composites for Hy- drogen Evolution under Sunlight}

\author{
WEI Jie ${ }^{1}$, LI Xue-Dong ${ }^{1}$, WANG Hong-Zhi ${ }^{1}$, ZHANG Qing-Hong ${ }^{2}$, LI Yao-Gang ${ }^{1}$
}

( 1. State Key Laboratory of Modification of Chemical Fibers and Polymer Materials, College of Materials Science and Engineering, Donghua University, Shanghai 201620, China 2. Engineering Research Center of Advanced Glasses Manufacturing Technology, College of Material Science and Engineering, Donghua University, Shanghai 201620, China)

\begin{abstract}
Using acetonitrile as the solvent and glucose as the raw material, nitrogen doped carbon quantum dots (NCDs) were prepared by the solvothermal method. The size of NCDs is around $4 \mathrm{~nm}$. The emission spectrum of NCDs showed red shift when the excitation wavelength of quantum dots increased from $330 \mathrm{~nm}$ to $470 \mathrm{~nm}$. Titanium dioxide was coated by NCDs by mixing a certain proportion of $\mathrm{NCDs} \mathrm{TiO}_{2}$ and ultrapure water through 60 min ultrasonic and aging under $80^{\circ} \mathrm{C}$ for $24 \mathrm{~h}$. NCDs/titanium dioxide composites show good photocatalyst performance as compared with pure NCDs and pure titanium dioxide, because the NCDs can expand the absorption spectrum and reduce the photogenerated electrons and holes. The $\mathrm{NCDs} / \mathrm{TiO}_{2}$ composites at the raw ratio of $m(\mathrm{NCDs}): m\left(\mathrm{TiO}_{2}\right)=15: 85$ show the best hydrogen evolution performance, using methanol as the sacrificial agent. The composite material displays good stability and certain photocatalytic performance after three cycles.
\end{abstract}

收稿日期: 2015-02-09; 收到修改稿日期：2015-05-18

基金项目: 国家自然科学基金(51172042); 高等学校学科创新引智计划资助(111-2-04); 高等学校博士学科点专项科研基金 (20110075130001); 教育部创新团队发展计划(IRT1221); 上海市科委项目(12nm0503900，13JC1400200); 中央 高校基本科研业务费

National Natural Science Foundation of China(51172042); Program of Introducing Talents of Discipline to Universities(111-2-04); Specialized Research Fund for the Doctoral Program of Higher Education(20110075130001); Innovative Research Team in University(IRT1221); Science and Technology Commission of Shanghai Municipality(12nm0503900, 13JC1400200); Shanghai Natural Science Foundation

作者简介: 魏 婕(1981-), 女, 博士研究生. E-mail: donghuaweijie@163.com

通讯作者: 王宏志, 教授. E-mail: wanghz@dhu.edu.cn 
Key words: nitrogen doped carbon quantum dots: nitrogen doped carbon quantum dots/titanium dioxide composite: hydrogen evolution under sunlight: solvothermal reaction

由于石油资源日益枯竭, 氢气作为一种重要的 清洁能源和化工原料受到人们广泛的重视。在众多 的氢气制备方法中, 使用半导体材料对水进行分解 反应是一种价格低廉且无污染的制备方法。1972 年, Fujishima 和 Honda ${ }^{[1]}$ 以水为原料采用电化学方法在 二氧化钛 $\left(\mathrm{TiO}_{2}\right)$ 电极的表面制备了 $\mathrm{H}_{2}$, 开启了半导 体制备氢气的先河。此后, $\mathrm{ZnO}$ 和 $\mathrm{WO}_{3}$ 等多种半导 体作为光催化材料先后成功实现了水分解制氢。在 众多半导体催化剂中, $\mathrm{TiO}_{2}$ 具有制氢效率高, 本身没 有毒性, 化学性能稳定, 制备成本低等优点, 得到广 泛关注。但是, 该材料存在着两方面的不足: (1) $\mathrm{TiO}_{2}$ 的带隙很宽 (以锐钛矿为例, 其带隙为 $3.2 \mathrm{eV}$ ), 只能 利用太阳光中的紫外光激发; (2) $\mathrm{TiO}_{2}$ 表面的电子和 空穴在催化过程中容易快速复合, 导致光量子效率 低 ${ }^{[2]}$ 。对于 $\mathrm{TiO}_{2}$ 的改性方法, 主要有半导体材料与 $\mathrm{TiO}_{2}$ 复合, 金属离子和非金属离子的掺杂, 贵金属 沉积等, 其中半导体材料与 $\mathrm{TiO}_{2}$ 复合一直是非常有 效的方法。

作为碳材料家族的新成员, 碳量子点具有独 特光学性能和电学性能、生物相容性好、比表面 积大和无毒等优点, 被广泛应用于光催化 ${ }^{[3]}$ 、检测 ${ }^{[4]}$ 和光学等领域 ${ }^{[5]}$ 。氮掺杂碳量子点是在碳量子点 的基础上发展起来的一种新材料, 由于氮原子的 引入, 改变了碳量子点的内部电子运输, 进而影 响了材料的各种性能。已有研究发现, 氮原子的 引入能够拓宽碳基量子点在可见光区域的吸收光 谱 ${ }^{[6]}$ 。使用氮掺杂的碳量子点与 $\mathrm{TiO}_{2}$ 复合, 更加 有利于体系在可见光区域的光吸收 ${ }^{[7]}$ 。本工作使 用水热法制备的 NCDs 能够吸收紫外光和可见光, 与 $\mathrm{TiO}_{2}$ 复合, 不仅拓宽了催化剂对太阳光的响应 范围, 而且能够有效抑制电子和空穴的复合, 提 高催化效率。

\section{1 实验方法}

\section{1 原料}

葡萄糖, 乙腈和二氯甲烷, 化学纯, 国药集 团化学试剂有限公司生产; $\mathrm{TiO}_{2}, \mathrm{AEROXIDE}$ $\mathrm{TiO}_{2} \mathrm{P} 25$, 赢创(EVONIK INDUSTRIES); 超纯水, 采用 PURIST 超纯水系统制备, RephiLe Bioscience, Ltd.。

\section{2 仪器与测试}

采用日本 JEOL 公司的(JEM-2100F 型)场发射透 射电子显微镜对所制备光催化剂的晶粒形貌和尺寸 进行微观分析, 加速电压为 $200 \mathrm{kV}$ 。采用美国 Thermo Fisher 生产的(Nicolet 6700)傅里叶变换红外光谱仪 测试样品的红外光谱。光谱范围: $7800 \sim 350 \mathrm{~cm}^{-1}$; 信噪比: $\geqslant 50000: 1$; 分辨率: $\leqslant 0.09 \mathrm{~cm}^{-1}$ 。采用日 本 JASCO 公司生产的( FP-6600)荧光光谱仪进行 发射光谱分析。采用美国 PerkinElmer 生产的 (Lambda 35) 紫外-吸收光谱仪测试样品的紫外-吸 收光谱。采用美国 Thermo Fisher 生产的 ESCALAB $250 X i$ 型 $X$ 射线光电子能谱仪测试样品的 $X$ 射线 光电子能谱。采用北京泊菲莱科技有限公司生产的 Labsolar 系列光催化设备 Labsolar-IIAG 进行光催 化制氢测试。采用 UV-1800 型紫外-可见分光光度 计(岛津仪器苏州有限公司)测定样品的紫外光谱。 采用北京泊菲莱科技有限公司生产的氙灯光源 PLS-SXE300/UV, 每次实验时将电流控制在 $15 \mathrm{~A}$ 。 氢气的气相色谱采用上海天美公司生产, 型号为 GC7900 的进行检测。

\subsection{NCDs 的制备}

用分析天平称取葡萄糖 $0.02 \mathrm{~g}$, 用量筒量取乙 腈溶液 $80 \mathrm{~mL}$, 分别放入容积为 $100 \mathrm{~mL}$ 的聚四氟 乙烯水热釜内胆中。随后, 把水热釜放入 $180^{\circ} \mathrm{C}$ 的 烘箱中水热反应 $8 \mathrm{~h}$ 。待水热釜冷却后, 取出内胆, 倒出反应液。用孔径为 $0.22 \mu \mathrm{m}$ 的微孔滤膜对反应 液进行抽滤。再用二氯甲烷对反应液进行反复洗涤 直到水溶液层完全透明为止。使用旋转蒸发仪蒸发 溶液直到溶液剩下 $5 \mathrm{~mL}$, 再将 $5 \mathrm{~mL}$ 浓缩液进行冷 冻干燥 $24 \mathrm{~h}$ 。待干燥结束得到的褐色粉末即为 NCDs。

\section{$1.4 \mathrm{NCDs} / \mathrm{TiO}_{2}$ 复合材料的制备}

按照投料比 $m(\mathrm{NCDs}): m\left(\mathrm{TiO}_{2}\right)$ 为 $5: 95,10: 90$, 15: 85, 20: 80 称量 $\mathrm{NCDs}$ 和 $\mathrm{TiO}_{2}$ 的混合物。随后, 将混合粉末分别添加到 $5 \mathrm{~mL}$ 超纯水中超声 $60 \mathrm{~min}$, 使之充分混合均匀。将超声后的混合液放在冰箱中 冷冻成块后送至真空冷冻机内冷冻干燥, 条件为 $-40^{\circ} \mathrm{C}, 24 \mathrm{~h}$; 最后, 将得到的粉末放在 $80^{\circ} \mathrm{C}$ 的烘箱 内处理 $24 \mathrm{~h}$ 。收集得到的复合材料粉末进行测试。

\section{5 光催化制氢性能的研究}

取 $1 \mathrm{~g} \mathrm{NCDs}$ 放入 $200 \mathrm{~mL}$ 超纯水中, 随后加入 $5 \mathrm{~mL}$ 甲醇作为光催化牺牲剂。在磁力搅拌下进行太阳光 
光催化制氢反应，反应 $1 \mathrm{~h}$ 和 $2 \mathrm{~h}$ 后取样采集氢气。 重复以上步骤测试纯的 $\mathrm{NCDs}, \mathrm{NCDs} / \mathrm{TiO}_{2}$ 复合材料 和纯的 $\mathrm{TiO}_{2}$ 的光催化制氢性能。

\section{2 结果与讨论}

\subsection{NCDs 的表征结果}

\subsection{1 显微结构分析}

图 1 为 NCDs 的透射电镜照片, 由图可以看出, 在乙腈溶剂中加入匍萄糖, 通过溶剂热法制备得到 的 NCDs 尺寸分布均匀, 粒径在 $4 \mathrm{~nm}$ 左右。从图 1 中还可以看出, 通过此方法制备的产物没有明显的 团聚现象, 这可能是由于 NCDs 表面含有亲水性基 团, 使其能够稳定分散在水溶液中。

\subsection{2 荧光和吸收光谱分析}

图 2 为 NCDs 的苂光激发和发射光谱图, NCDs 的激发光谱显示, $400 \mathrm{~nm}$ 左右的激发光可以激发出

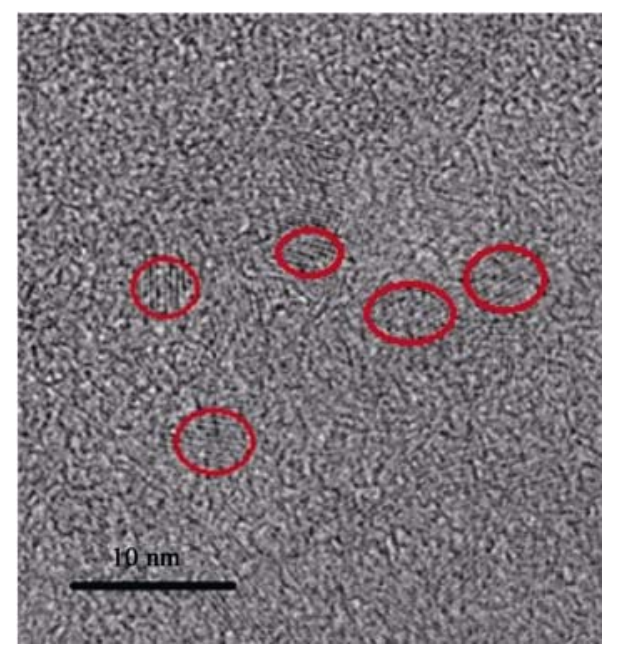

图 1 NCDs 的 TEM 照片

Fig. 1 TEM image of nitrogen doped carbon quantum dots

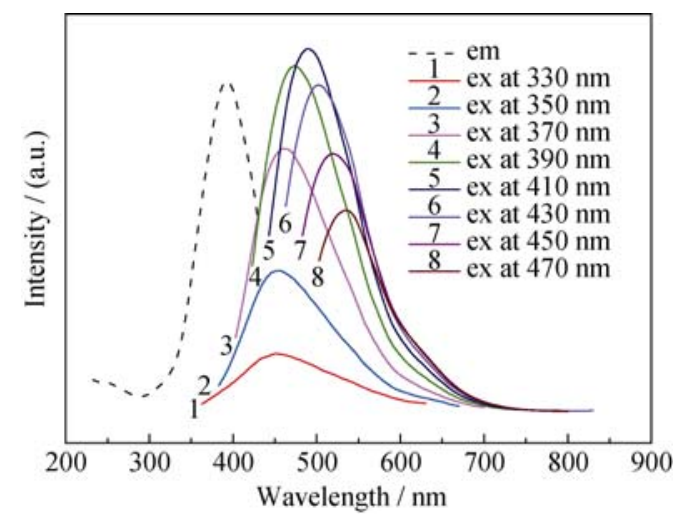

图 2 NCDs 的苂光激发和发射光谱图

Fig. 2 Fluorescence emission and excitation spectra of nitrogen doped carbon quantum dots
最强的菼光光谱。随着激发波长从 $330 \mathrm{~nm}$ 增加到 $470 \mathrm{~nm}, \mathrm{NCDs}$ 的发射光谱出现了红移, 该现象说明 NCDs 可以被比较宽的激发光所激发。

图 3 为 NCDs 的紫外吸收光谱, 插图分别为在 日光灯下的 NCDs 的水溶液和 $365 \mathrm{~nm}$ 紫外光激发下 显示出蓝色荧光的 NCDs 的水溶液。从图 3 可以看 出, NCDs 从 $200 \mathrm{~nm}$ 到 $800 \mathrm{~nm}$ 均有吸收, 包括了可 见光部分和紫外光部分, 这有利于更好地利用太阳 光进行光催化制氢。紫外吸收光谱在 $280 \mathrm{~nm}$ 左右非 常强的吸收是典型的芳香族 $\pi$ 体系吸收 ${ }^{[8]}$ 。

\subsection{3 红外光谱分析}

图 4 为 NCDs 的红外光谱图, 在 $1051 \mathrm{~cm}^{-1}$ 的 波峰为环氧官能团的伸缩震动; $1633 \mathrm{~cm}^{-1}$ 的波峰为 苯环的 $\mathrm{C}=\mathrm{C}$ 键的震动, 正好对应紫外吸收光谱, 证 明有大 $\pi$ 键存在。 $1138 \mathrm{~cm}^{-1}$ 的波峰是 $\mathrm{C}-\mathrm{N}$ 键引起的, 由于葡萄糖本身不含有氮元素, $\mathrm{C}-\mathrm{N}$ 键的存在证明 乙腈参与了化学反应。3423 $\mathrm{cm}^{-1}$ 的波峰对应于 $\mathrm{O}-\mathrm{H}$ 的伸缩震动, 氮掺杂石墨烯量子点具有良好的亲水 性正是 O-H 所赋予的。 $1402 \mathrm{~cm}^{-1}$ 的波峰对应 O-H 的变形震动 ${ }^{[9-10]}$ 。

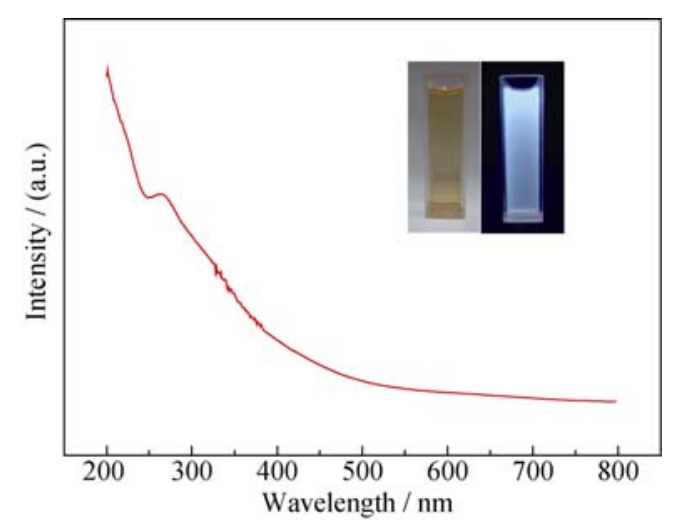

图 3 NCDs 的紫外吸收光谱

Fig. 3 Ultraviolet absorption spectrum of NCDs Inset shows the NCDs solution under daylight lamp and $365 \mathrm{~nm}$ excitation

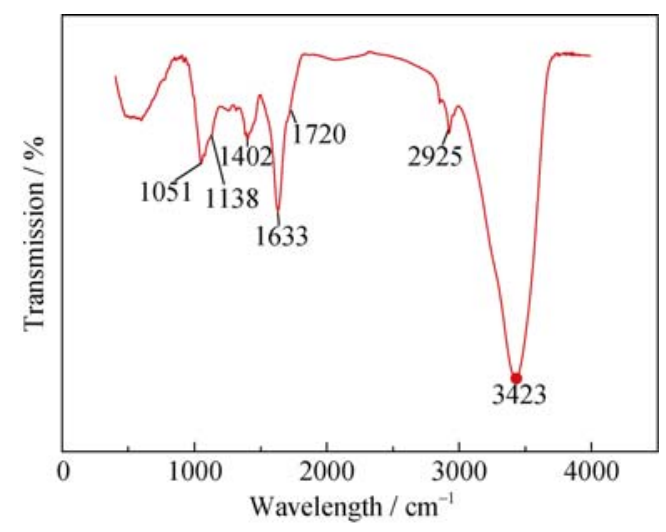

图 4 NCDs 的红外光谱图

Fig. 4 Infrared spectrum of NCDs 


\subsubsection{X 射线光电子能谱分析}

图 5(a)为 NCDs 的 X 射线光电子能谱全谱。从 图谱中可以看出, NCDs 含有氮、氧和碳三种元素。 碳元素对应于 $282.6 \mathrm{eV}$ 的峰, 氮元素对应于 $397 \mathrm{eV}$ 的峰, 氧元素对应于 $531 \mathrm{eV}$ 的峰。图 5(b)为 NCDs 的高分辨率 C1s 谱, 该图谱中的峰 284.2、285.5 $286.2 、 287.4 、 288.2 \mathrm{eV}$ 分别对应于 C-C、C-N、C-O、 $\mathrm{C}=\mathrm{O} 、 \mathrm{O}-\mathrm{C}=\mathrm{O}^{[11]}$ 。图 5(c)为 NCDs 的高分辨率 $\mathrm{N} 1 \mathrm{~s}$ 谱，图谱中的波峰对应于三种不同形式的掺杂氮， 398.50、399.20 和 $400.60 \mathrm{eV}$ 分别对应于氮原子替代 吡啶形状碳环中的碳原子(pyridine-like $\mathrm{N}$ atom)、氮
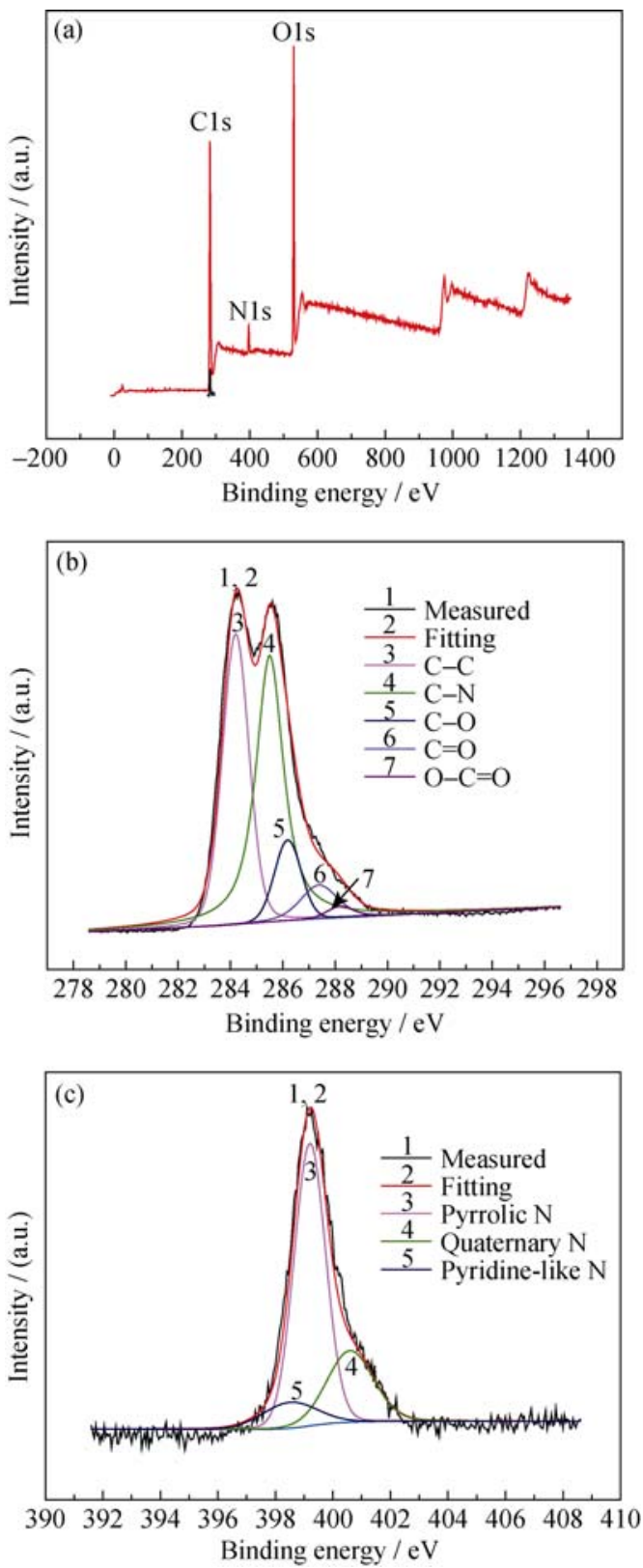

图 5 NCDs 的 X 射线光电子能谱

Fig. 5 XPS spectra of NCDs

(a) Full scan spectrum, (b) C1s and (c) N1s 原子替代吹咯形状的碳结构中的碳原子(pyrrolic $\mathrm{N}$ atom) 和氮原子替代四元环结构中的碳原子 (quaternary $\mathrm{N}$ atom) ${ }^{[12]}$ 。

\section{$2.2 \mathrm{NCDs} / \mathrm{TiO}_{2}$ 复合材料的表征结果}

\subsection{1 显微结构分析}

图 6 为 $\mathrm{NCDs} / \mathrm{TiO}_{2}$ 复合材料的高分辨透射电子 显微镜照片。通过测量晶面间距可以发现, 球形材 料的中心部分的晶面间距为 $0.351 \mathrm{~nm}$, 对应于 $\mathrm{TiO}_{2}$ 的(101)晶面。在 $\mathrm{TiO}_{2}$ 的表面包覆着一层晶面间距为 $0.263 \mathrm{~nm}$ 的 NCDs。从高分辨透射电镜照片中可以 看出 $\mathrm{NCDs}$ 包裹在 $\mathrm{TiO}_{2}(\mathrm{P} 25)$ 表面。同时, 从复合材 料的 SEM 照片(图 7)中也可以看到 NCDs 包裹在 $\mathrm{TiO}_{2}$ 表面。

\subsection{2 紫外-可见吸收光谱}

图 8 为 $\mathrm{NCDs} / \mathrm{TiO}_{2}$ 复合材料和纯的 $\mathrm{TiO}_{2}$ 的紫外可见吸收光谱图, 从图中可以看出, 纯的 $\mathrm{TiO}_{2}(\mathrm{P} 25)$ 在 $400 \mathrm{~nm}$ 之前没有吸收; $\mathrm{TiO}_{2}$ 与 $\mathrm{NCDs}$ 复合后, 光吸收

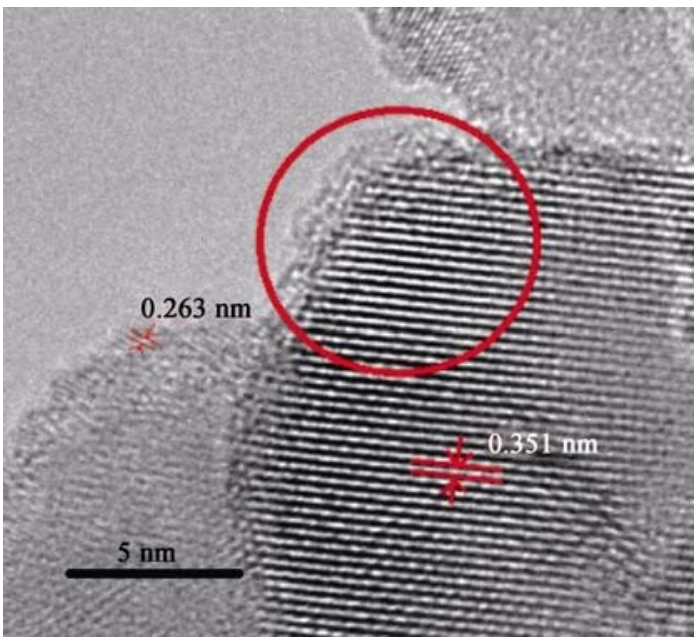

图 $6 \mathrm{NCDs} / \mathrm{TiO}_{2}$ 复合材料的高分辨透射电镜照片

Fig. 6 HRTEM image of $\mathrm{NCDs} / \mathrm{TiO}_{2}$ composite

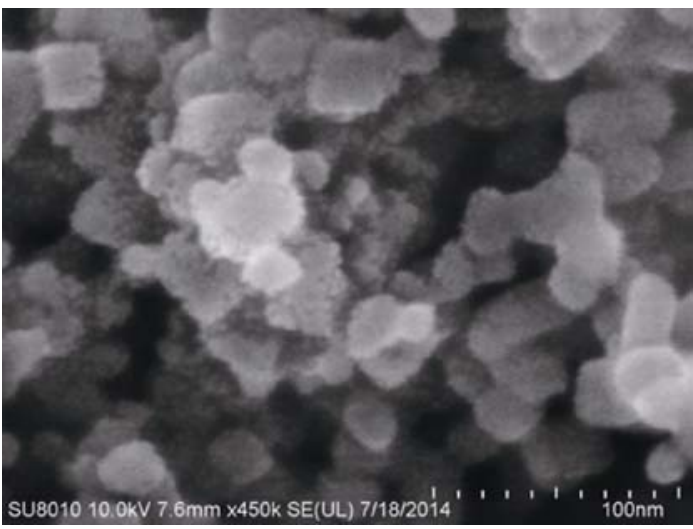

图 $7 \mathrm{NCDs} / \mathrm{TiO}_{2}$ 复合材料的扫描电镜照片

Fig. 7 SEM image of $\mathrm{NCDs} / \mathrm{TiO}_{2}$ composite 


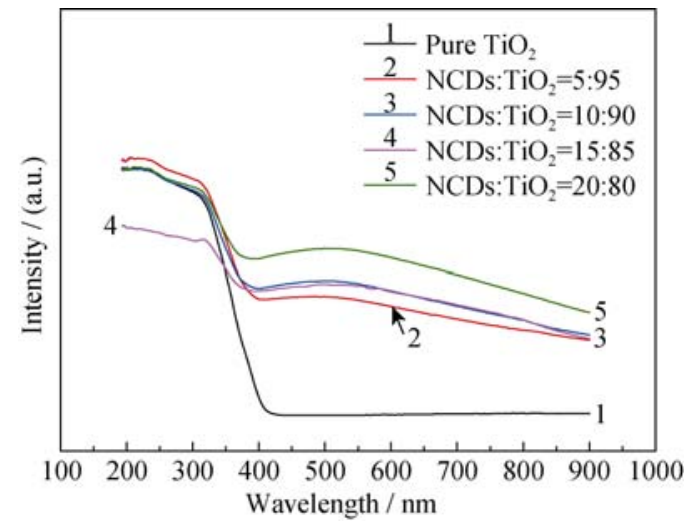

图 $8 \mathrm{NCDs} / \mathrm{TiO}_{2}$ 复合材料和纯的 $\mathrm{TiO}_{2}$ 的紫外-可见吸收光谱图 Fig. 8 UV-visible absorption spectra of $\mathrm{NCDs} / \mathrm{TiO}_{2}$ composite and pure $\mathrm{TiO}_{2}$

从 $800 \mathrm{~nm}$ 开始, 整个体系的光吸收被拓宽, 有利于 光催化制氢反应的进行。随着 NCDs 含量的增加, 体 系的光吸收强度增大。

\subsection{3 荧光光谱分析}

从图 9 可以看出, 随着复合材料中 NCDs 含量 的减少, 材料的苂光光谱强度也在下降。说明复合 材料中的光生电子的空穴对被抑制, 复合材料将表 现出比 $\mathrm{NCDs}$ 和 $\mathrm{TiO}_{2}$ 更好的光催化制氢性能。

从以上分析结果可以推断 $\mathrm{NCDs} / \mathrm{TiO}_{2}$ 复合材料 光催化制氢可能的机理为: 在光的激发下, 电子从 $\mathrm{TiO}_{2}$ 的价带被跃迁到导带; 然后电子从 $\mathrm{TiO}_{2}$ 和 NCDs 的接触面迁移至 NCDs; 随后, 在 NCDs 表面 的活性中心进行水的还原制氢。在整个反应的过程 中, NCDs 除了作为反应的活性中心, 本身也能够吸 收可见光和紫外光进行光催化制氢反应。

\subsection{4 太阳光催化制氢分析}

图 10 为投料比为 $m(\mathrm{NCDs}): m\left(\mathrm{TiO}_{2}\right)=15: 85$ 的

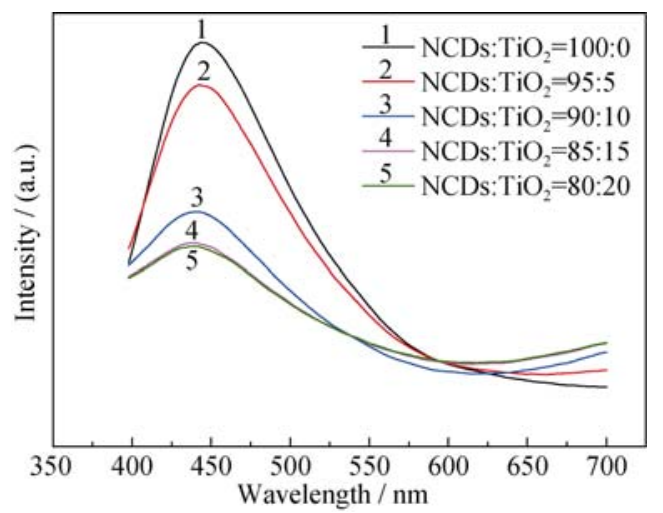

图 $9 \mathrm{NCDs} / \mathrm{TiO}_{2}$ 复合材料在室温下的荧光光谱, 激发波长为 $365 \mathrm{~nm}$

Fig. 9 Room temperature photoluminescence spectra of $\mathrm{NCDs} / \mathrm{TiO}_{2}$ composite excited by the light with wavelength of $365 \mathrm{~nm}$

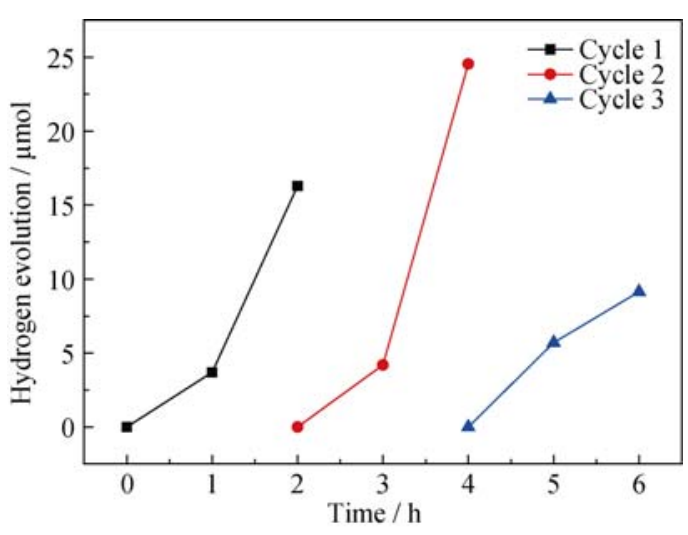

图 10 投料比为 $m(\mathrm{NCDs}): m\left(\mathrm{TiO}_{2}\right)=15: 85$ 的条件下制备的复 合材料产生氢气的稳定性研究

Fig. 10 Stability study of photocatalytic time course of hydrogen production for the composites prepared at weight ratio of $m$ (NCDs): $m\left(\mathrm{TiO}_{2}\right)=15: 85$

The black line indicates using photocatalysis to produce hydrogen for the first time. The red line indicates the second time, and the blue line indicates the third time

条件下制备的复合材料产生的氢气与反应时间的 关系，从图中可以看出：随着反应的进行，催化反 应进行 $2 \mathrm{~h}$ 生产氢气的量明显高于 $1 \mathrm{~h}$ 生产氢气的 量, 说明催化反应有一个活化过程。在回收第一次 经催化反应的催化剂后, 重新进行第二次反应, $2 \mathrm{~h}$ 的催化效果明显比第一次催化的效果好, 但是随 着进行第三次催化反应，催化效果比第二次差，催 化剂颜色变淡。主要是由于包裹在 $\mathrm{TiO}_{2}$ 表面的褐 色 NCDs 随着反应的进行, 表面的生色官能团即催 化制氢的活化官能团随着反应的进行逐渐被消耗 造成的。

图 11 为纯的 NCDs、 $\mathrm{NCDs} / \mathrm{TiO}_{2}$ 复合材料和纯 的 $\mathrm{TiO}_{2}$ 样品的光催化制氢活性。(这些数据是

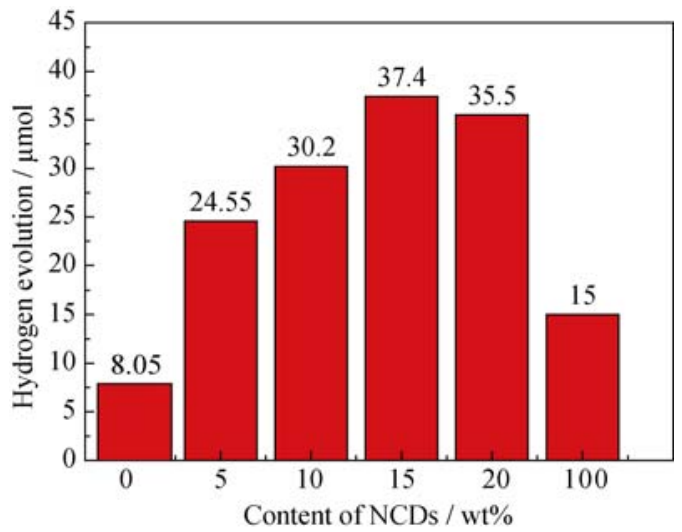

图 11 纯的 $\mathrm{NCDs}, \mathrm{NCDs} / \mathrm{TiO}_{2}$ 复合材料和纯的 $\mathrm{TiO}_{2}$ 在太阳光 下光催化制氢活性

Fig. 11 Comparison of the photocatalytic hydrogen production activities of pure NCDs, pure $\mathrm{TiO}_{2}$ and NCDs/ $\mathrm{TiO}_{2}$ compound under sunlight 
第二次循环使用催化剂时，体系反应 $2 \mathrm{~h}$ 产生氢气 的量)。从图 11 可以看出: 纯的 NCDs 和纯的 $\mathrm{TiO}_{2}$ 的光催化制氢效果都小于 $\mathrm{NCDs} / \mathrm{TiO}_{2}$ 复合材料, 主 要原因为两者的复合减少了光生电子和空穴复合的 机会; 同时, NCDs 能够有效拓宽本来只能吸收紫外 光的 $\mathrm{TiO}_{2}$ 的吸收光谱。复合材料的光催化性能随着 NCDs 的增加而增强, 当投料比为 $m(\mathrm{NCDs}): m\left(\mathrm{TiO}_{2}\right)=$ 15: 85 时, 体系的制氢量最高, 为 $37.4 \mu \mathrm{mol}$ 。当投料 比为 $m(\mathrm{NCDs}): m\left(\mathrm{TiO}_{2}\right)=20: 80$ 时, 体系的光催化制 氢性能不再提高。可能的原因为: 体系中 NCDs 具 有不透明性, 并且造成光散射, 从而降低了复合材 料内部颗粒对光的吸收; 此时整个体系减少的光吸 收的量抵消了因为 NCDs 的增加而多吸收的光, 致 使光催化性能降低 ${ }^{[13]}$ 。

\section{3 结论}

采用溶剂热法, 将葡萄糖放入乙腈溶剂中合成 了 NCDs，方法简单易操作，制备的 NCDs 颗粒分布 均匀, 能够稳定地分散在水溶液中。同时, 使用物理 混合并且陈化的方法制备了 $\mathrm{NCDs} / \mathrm{TiO}_{2}$ 复合材料。 由于 NCDs 能够同时吸收紫外光和可见光, 有效地 拓宽了材料光吸收的范围; 此外, 复合材料能够有 效地阻止空穴与电子的复合, 很大程度上提高了光 催化效率。随着 NCDs 在复合材料中含量的增加, 光 催化制氢性能也随着提高。当投料比为 $m(\mathrm{NCDs})$ : $m\left(\mathrm{TiO}_{2}\right)=15: 85$ 时, 催化效果最佳。但由于复合材料 表面被过量的 NCDs 包覆会影响内部颗粒对于光的 吸收，以及造成了光的散射，当投料比为 $m(\mathrm{NCDs})$ : $m\left(\mathrm{TiO}_{2}\right)=20: 80$ 时, 复合材料的光催化制氢性能有 所下降。实验所获得的材料不但没有毒性, 而且原 料简单易得，可有效提高光催化制氢性能。

\section{参考文献:}

[1] FUJISHIMA A, HONDA K. Electrochemical photolysis of water at a semiconductor electrode. Nature, 1972, 238: 37-38.

[2] YU Z M, MENG J L, XIAO J R, et al. Cobalt sulfide quantum dots modified $\mathrm{TiO}_{2}$ nanoparticles for efficient photocatalytic hydrogen. Int. J. Hydrogen Energy, 2014, 39: 15387-15393.

[3] CHEN F, ZOU W W, QU W W, et al. Photocatalytic performance of a visible light $\mathrm{TiO}_{2}$ photocatalyst prepared by a surface chemical modification process. Catalysis Communications, 2009, 10: 15101513

[4] LIU Q L, XU S H, NIU C X, et al. Distinguish cancer cells based on targeting turn-on fluorescence imaging by folate functionalized green emitting carbon dots. Biosensors \& Bioelectronics, 2015, 64: 119-125.

[5] CHEN J, LIU W, MAO L H, et al. Synthesis of silica-based carbon dot/nanocrystal hybrids toward white LEDs. Journal of Materials Science, 2014, 49(21): 7391-7398.

[6] YEH T, CHEN S, YEH C, et al. Tuning the electronic structure of graphite oxide through ammonia treatment for photocatalytic generation of $\mathrm{H}_{2}$ and $\mathrm{O}_{2}$ from water splitting. The Journal of Physical Chemistry, 2013, 117: 6516-6524.

[7] ZHANG Y Q, MA D K, ZHANG Y G, et al. N-doped carbon quantum dots for $\mathrm{TiO}_{2}$-based photocatalysts and dye-sensitized solar cells. Nano Energy, 2013, 2: 545-552.

[8] RAN C X, WANG M Q, GAO W Y, et al. A general route to enhance the fluorescence of graphene quantum dots by Ag nanoparticles. RSC Adv., 2014, 4: 21772-21776.

[9] MA Z, MING H, HUANG H, et al. One-step ultrasonic synthesis of fluorescent $\mathrm{N}$-doped carbon dots from glucose and their visiblelight sensitive photocatalytic ability. New J. Chem., 2012, 36(4): 861-864.

[10] HU S L, NIU K Y, SUN J, et al. One-step synthesis of fluorescent carbon nanoparticles by laser irradiation. J. Mater. Chem., 2009, 19(4): 484-488.

[11] LI Y, ZHAO Y, CHENG H, et al. Nitrogen-doped graphene quantum dots with oxygen-rich functional groups. J. Am. Chem. Soc., 2012, 134(1): 15-18.

[12] LI Y, HU Y, ZHAO Y, et al. An electrochemical avenue to green-luminescent graphene quantum dots as potential electronacceptors for photovoltaics. Adv. Mater., 2011, 23(6): 776-780.

[13] XIANG Q J, YUJ G, JARONIEC M. Enhanced photocatalytic $\mathrm{H}_{2}$-production activity of grapheme-modified titaniananosheets. Nanoscale, 2011, 3: 3670-3678. 